\title{
Catálogo actualizado de las plantas vasculares del Parque Nacional Isla del Coco, Costa Rica
}

\author{
Armando Estrada-Chavarría ${ }^{1 *}$, Joaquín Sánchez-González² \& Alexander Rodríguez-González ${ }^{3}$ \\ 1. Departamento de Historia Natural, Museo Nacional de Costa Rica, San José, Costa Rica; \\ aestrada@museocostarica.go.cr, jsanchez@museocostarica.go.cr, arodriguez@museocostarica.go.cr \\ * Correspondence
}

Recibido 30-I-2019. Corregido 08-VI-2019. Aceptado 20-VIII-2019.

\begin{abstract}
Updated catalog of the vascular plants of Isla del Coco National Park. Introduction: The flora of Isla del Coco has been the subject of various collection studies and publications efforts for more than 300 years. During these years 263 species were registered; however, there have been nomenclature and classification changes, and an increase of known species since the last publication. Objective: To prepare an updated catalog of the vascular plants of Isla del Coco. Methods: The catalog present here is based on the review of herbarium specimens, publications, and plants collected between 2016 and 2017 by the authors. An updated list of plants on the island was prepared. In addition, the estimated richness and the quality of the inventory were determined through biodiversity estimators and the proportion of species according to their form, epiphytic habitat, and origin. Results: 296 species of vascular plants were registered, corresponding to 199 genera and 82 families, which represents about $80 \%$ of the expected diversity according to the estimators used (Chao- 1 and Clench equation). Of the total, $219(74 \%)$ species are native and $77(26 \%)$ are introduced. Native species include angiosperms (53\%), ferns (44\%) and lycophytes (3\%), and those introduced are represented only by angiosperms. The most diverse families and genera and the most common or abundant species are indicated. From the native flora, 48 spp. $(22 \%)$ are endemic, and is mainly composed of ferns $(58 \%)$. The flora has a high proportion of herbaceous species $(63 \%)$ and a low representation of arborescent species $(12 \%)$ and vines $(8 \%)$. There is also a high proportion of epiphytes ( $20 \%$ of the total flora), mostly composed of ferns $(69 \%)$. This study added 45 new species records to the island, of which 16 are new species for science and endemic to the island, and eight are introduced species. Thirty-six species names were updated or corrected compared to Trusty et al. (2006). A catalog of species with annotations is provided. Conclusions: The flora of Isla del Coco presents certain peculiarities that distinguish it from other Pacific oceanic islands, especially due to the high proportion of endemic ferns, and the high diversity of this group in general, and the relatively low percentage of endemism. Also, characteristics as the low diversity of tree species, with high dominance of Sacoglottis holdridgei in all the forests of the island, and the high proportion of epiphytic species, mainly composed of ferns. The proportion of introduced species is relatively low, compared to other oceanic Pacific islands.
\end{abstract}

Key words: vascular plants, floristic diversity, oceanic islands, Cocos Island, Eastern Tropical Pacific.

Estrada-Chavaría, A., Sánchez-González. J. \& Rodríguez-González, A. (2020). Catálogo actualizado de las plantas vasculares del Parque Nacional Isla del Coco, Costa Rica.. Revista de Biología Tropical, 68(Supl. 1), S73-S88.

La Isla del Coco es una isla oceánica de origen volcánico que pertenece a Costa Rica. Se localiza en las coordenadas $5^{\circ} 31^{\prime} 47^{\prime \prime} \mathrm{N}$ y 8703'37' W, a una distancia de $496 \mathrm{~km}$ de Cabo Blanco en Costa Rica, 1049 km de Cabo
Corrientes en Colombia, $932 \mathrm{~km}$ de Cabo San Francisco, Ecuador, y a $681 \mathrm{~km}$ de la Isla Pinta, Islas Galápagos, Ecuador. Posee una extensión geográfica de $24 \mathrm{~km}^{2}$ y la mayor altitud es el Cerro Iglesias con 575 m (Instituto Geográfico 
Nacional de Costa Rica, 2000). Esta isla fue descubierta en 1526 por el navegante español Juan Cabezas. Posteriormente fue utilizada como sitio de refugio y abastecimiento de agua por balleneros y piratas.

Es posible que las primeras recolectas botánicas del territorio costarricense se realizaran en la Isla del Coco por el bucanero y naturalista británico William Dampier, cuando se detuvo en esta isla en 1684 (Hammel, Grayum, Herrera, \& Zamora, 2004), aunque en el relato de su viaje, solo hace una breve descripción de la isla, en la que menciona la abundancia de palmas de coco y una hierba llamada gramadael por los españoles (sic) (Dampier, 1699). Posteriormente el médico y botánico británico Archibald Menzies, en 1794 y/o 1795, desembarcó en la Isla del Coco y recolectó unas pocas muestras que aún se preservan (Hammel et al., 2004).

En 1838 George W. Barclay, botánico que acompañó al capitán Edward Belcher en el viaje del HMS Sulphur al Pacífico, realizó las primeras recolectas bien documentadas de plantas de la Isla del Coco (Belcher, 1843), este botánico resaltó que esta isla es más notable por la exuberancia de su vegetación que por su riqueza y variedad. Posteriormente, Bentham (1844) publicó nueve especies nuevas producto de la estadía de esta expedición en la isla. Más tarde, en 1891, Alexander Agassiz recolectó algunas plantas y posteriormente Rose (1892) publicó una lista de apenas seis taxones, dos de ellos géneros. Aun así, puede considerarse que esa es la primera lista publicada de plantas de la Isla del Coco.

La primera expedición financiada por el gobierno de Costa Rica fue realizada por Anastasio Alfaro, Henri Pittier y Paul Biolley en 1898. En el informe Pittier registró 36 taxones a nivel de familia o mayores, de los cuales 30 pertenecen a angiospermas y los otros a helechos, musgos, líquenes, algas y hongos. Allí identificó 11 especies y 14 géneros; además, aportó comentarios taxonómicos, morfológicos y ecológicos para cada una de las entidades registradas (Pittier, 1899). En 1899 Robert E. Snodgrass y Edmund Heller, naturalistas a bordo de la expedición Hopkins-Stanford, también recolectaron plantas en la Isla del Coco. En 1902 se publicó una lista que incluye 15 especies de angiospermas y 16 de helechos y licófitos, además de seis especies de briófitos (Robinson, 1902). En este mismo año, Pittier visitó de nuevo la isla. Tanto de la primera como de esta expedición se conservan 72 ejemplares en el Herbario Nacional de Costa Rica. En 1905 la Academia de Ciencias de California organizó una expedición a las islas Galápagos, cuyo botánico fue Alban Stewart. En su viaje se detuvieron diez días en la Isla del Coco y Stewart hizo recolección de plantas, de las cuales identificó 68 especies y siete a nivel de género o familia. Además, en su informe incluyó anotaciones taxonómicas y de distribución conocida de cada especie (Stewart, 1912). En 1930 se realizó una expedición a las islas Galápagos y la Isla del Coco a cargo de Vincent Astor con el botánico Henry K. Svenson. En la Isla del Coco permanecieron tres días. De esta expedición existen dos publicaciones: una donde se registran cuatro especies de musgos y 27 de angiospermas (Svenson, 1935), y en la otra se informa de 22 especies de helechos (Svenson, 1938).

La primera recopilación sistemática de plantas que se publicó es la de Fosberg \& Klawe (1966), la cual está basada en recolectas que el segundo autor realizó en 1964 y de una revisión de literatura disponible a la fecha. La lista incluye 142 especies identificadas y seis a nivel de género. Además de angiospermas, abarca musgos, hepáticas, hongos y líquenes. Sin embargo, esta lista carece de especímenes testigo de las especies mencionadas. En 1975, Luis Diego Gómez, del Museo Nacional de Costa Rica, informó la presencia de 60 especies de pteridófitos y licófitos en la isla; además incluyó información sobre la distribución geográfica de las especies (Gómez, 1975a). En otra publicación del mismo año presentó descripciones diagnósticas de cada una de las 60 especies, notas de ecología y citó especímenes examinados en diferentes herbarios (Gómez, 1975b). 
A partir de la creación del Parque Nacional Isla del Coco (Decreto Ejecutivo 8748, 1978), la recolección y documentación de la flora de esta isla se incrementó considerablemente, sobre todo, por parte de investigadores costarricenses pertenecientes al Museo Nacional, Instituto Nacional de Biodiversidad y universidades estatales. En años recientes aparecen nuevas publicaciones sobre la flora vascular de la Isla del Coco. Rojas \& Trusty (2004) anotaron 80 especies de pteridófitos, de las cuales 18 son endémicas $(22,5 \%)$. Además, incluyeron aclaraciones sobre nuevas distribuciones, diferentes morfologías y confusiones taxonómicas pasadas. Trusty, Kesler, \& Delgado (2006) publicaron la flora vascular más completa de la Isla del Coco, basándose en recolectas propias de los autores y en la revisión de material de diversos herbarios, registraron 263 especies de plantas vasculares, de las cuales 37 son endémicas. Dicho trabajo registró 51 especies consideradas nuevas para la flora de la isla; consideró, además, que el $42 \%$ de la flora vascular nativa está compuesta por helechos y que el $50 \%$ de las especies endémicas de esta isla pertenecen a este grupo de plantas. Adicionalmente, Trusty et al. (2006) describieron los tipos de vegetación y aportaron suficiente información sobre otros aspectos físicos, históricos y biológicos. Finalmente, Bogarín, Warner, Powell, \& Savolainem (2011), describieron la flora orquideológica de la isla. Se trata de cinco especies, con descripciones, ilustraciones y aportes de la historia natural de cada una.

Durante los años 2016 al 2018 el Museo Nacional de Costa Rica llevó a cabo un proyecto para documentar las plantas, hongos, líquenes y aves en la Isla del Coco, con el fin de crear un sitio en internet sobre esta parte de la biodiversidad terrestre de la isla. Se determinó que se han presentado muchos cambios nomenclaturales, de clasificación y de incremento de especies desde el último trabajo realizado (Trusty et al., 2006), por lo que se considera necesaria la publicación de una lista de especies actualizada de plantas de la isla. El presente trabajo tiene como objetivo la presentación de un catálogo actualizado de las plantas vasculares de la Isla del Coco. Asimismo, se incluye una relación de sinonimias entre los nombres de plantas que han sido utilizados para la flora de esta isla desde 1892.

\section{MATERIAL Y MÉTODOS}

Este catálogo actualizado de las plantas vasculares de la Isla del Coco, se realizó mediante la búsqueda y revisión de especímenes recolectados en la Isla del Coco, depositados mayoritariamente en el Herbario Nacional de Costa Rica (CR) y en menor medida en los siguientes herbarios: Herbario de California Academy Sciences (CAS), Herbario de Field Museum (F), Herbario de Fairchild Tropical Botanic Garden (FTG), Herbario de Missouri Botanical Garden (MO) y Herbario de la Universidad de Costa Rica (USJ).

Adicionalmente, los autores realizaron tres giras de campo a la isla entre el 2016 y 2017, en los meses de junio, noviembre y diciembre, para recolectar y fotografiar especímenes. Además, se hizo una búsqueda y revisión de publicaciones previas de listas de plantas de la Isla del Coco. Cada nombre incluido en esas listas fue revisado para determinar su estatus taxonómico actual y su correspondencia respectiva con los nombres incluidos en el este catálogo. Los nombres científicos procedentes de las listas, que no poseen especímenes testigo o que no poseen relación taxonómica con los nombres actuales, se excluyeron del catálogo, pero se mencionan en los resultados como nombres dudosos o inciertos. También se excluyeron los nombres de plantas introducidas que, con certeza, actualmente no se observan ni registran en la isla. La lista de plantas sigue la clasificación taxonómica del Manual de Plantas de Costa Rica (Hammel, Grayum, Herrera \& Zamora, 2003a; 2003b; 2007; 2010; 2014; 2015); los cambios más recientes siguen la base de datos Tropicos de Missouri Botanical Garden (www. tropicos.org), basada a su vez en las propuestas del grupo para la filogenia de las angiospermas (APG IV, 2016).

Se estimó la diversidad máxima esperada con la ecuación de Clench y el índice 
no-paramétrico Chao-1 (Soberón \& Llorente, 1993, Jiménez-Valverde \& Hortal, 2003). La ecuación de Clench se calcula con la siguiente fórmula: $\mathrm{Sn}=\mathrm{a}^{*} \mathrm{n} /\left(1+\mathrm{b}^{*} \mathrm{n}\right)$, donde, $\mathrm{a}=$ tasa de incremento de nuevas especies al comienzo del inventario, $\mathrm{b}=$ parámetro relacionado con la forma de la curva, $\mathrm{n}=$ unidades de muestreo. Esta ecuación se ajustó mediante estimación no lineal, utilizando el método "Simplex and quasi-Newton" implementado en el programa STATISTICA y aleatorizando los datos con el programa EstimateS (Colwell 1997). El índice Chao-1 se calculó con la fórmula: Schao $=$ Sobs $+\mathrm{F}_{1}{ }^{2} /\left(2\left(\mathrm{~F}_{2}+1\right)\right)-\mathrm{F}_{1} \mathrm{~F}_{2} /\left(2\left(\mathrm{~F}_{2}+1\right)^{2}\right)$, donde, Sobs. $=$ número total de especies observadas; $\mathrm{F}_{1}$ : número de especies con un registro; $\mathrm{F}_{2}$ : número de especies con dos registros.

La diversidad de plantas de la isla se describió en función de su forma (árboles, arbustos, hierbas, bejucos y enredaderas), basado en el concepto de "formas de vida" de las plantas propuesto por Nivia Ruíz \& Cascante Marín (2008). Para cada forma se describe la contribución de los grupos taxonómicos más importantes. Además, se describe la representación del componente epífito en la composición florística de la isla.

En el apéndice se incluye el catálogo actualizado de plantas vasculares de la Isla del Coco ordenado por la categoría de división: Lycophyta, Monilophyta y Spermatophyta. Dentro de cada uno de estos grupos se ordenan familias, géneros y especies alfabéticamente. Para cada especie se indica: la autoría, sinonimias (Sin.) o nombres mal aplicados (=) publicados en listas previas, espécimen testigo, acrónimo de herbario donde está depositado, condición endémica (End.) o introducida (Introd.) en la isla, cuando aplica; número de ejemplares recopilados $(\mathrm{Ej}$.) y referencias de listas previas en que la especie o el género fue incluido (números superíndices).

La clasificación de especies introducidas en la isla, se basa en Trusty et al. (2006), en donde se considera introducida accidentalmente por humanos, cualquier especie ruderal encontrada por primera vez después de 1905, fecha de la primera colección de plantas bastante completa, realizada y publicada por Stewart (1912), a menos que haya evidencia de lo contrario. La condición de endemismo se determinó de acuerdo a lo indicado en el Manual de Plantas de Costa Rica (Hammel et al., 2003a, 2003b, 2007, 2010, 2014, 2015) y la base de datos Tropicos ya referida.

\section{RESULTADOS Y DISCUSIÓN}

Datos recopilados: La información florística recopilada de la Isla del Coco incluye un total de 1782 registros de plantas, provenientes principalmente de ejemplares del Herbario Nacional de Costa Rica (CR, 95\% de los registros) y en menor grado de otros herbarios (MO: 42 registros, FTG: 15, F: 11, CAS: 2, USJ: 2). Adicionalmente se incluyeron ocho registros de observaciones de campo. Este acervo de información es producto del esfuerzo y aporte de 44 recolectores de plantas, entre los que destacan por el número de ejemplares recolectados: Alexander Rojas Alvarado (265 ejemplares), Jennifer Trusty (264), Luis Diego Gómez Pignataro (224), Javier F. Quesada (125), Armando Estrada (118), Alexánder Rodríguez (79), José A. González (75), Alfonso Jiménez (74), Henri F. Pittier (73), Jorge Gómez-Laurito (55) y Eduardo Lépiz (54). Los registros más antiguos recopilados son ejemplares recolectados por George W. Barclay, R. E. Snodgrass y Henry F. Pittier, en 1838, 1889 y 1898, respectivamente. En cuanto a su distribución en el tiempo, la mayoría de los registros recopilados son recientes, un $60 \%$ fueron recolectados en las últimas tres décadas (1990 en adelante), producto principalmente de proyectos florísticos nacionales realizados por el Museo Nacional de Costa Rica y el Instituto Nacional de Biodiversidad, así como del estudio de Trusty et al. (2006); un $33 \%$ de los registros datan de 1960 a 1990 y un $7 \%$ fueron recolectados antes de 1960 (Fig. 1).

Aunque la isla ha sido recorrida ampliamente y se tienen registros de muchos sectores, la distribución del esfuerzo de recolecta de plantas es muy heterogénea (Fig. 2); se ha concentrado principalmente en los sitios de mayor accesibilidad, ubicados en el sector norte de la 


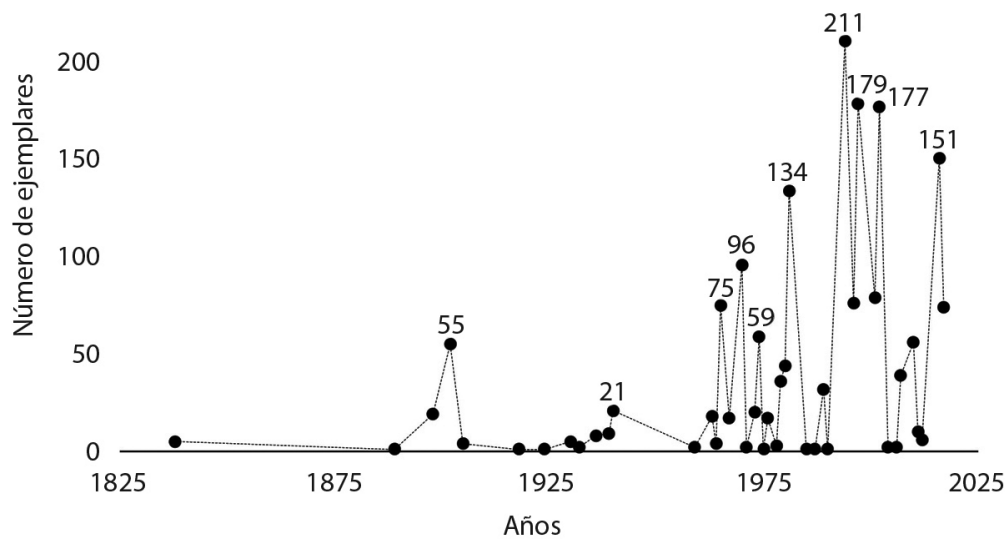

Fig. 1. Distribución en el tiempo de los eventos de recolecta de plantas vasculares recopilados para la Isla del Coco, Puntarenas, Costa Rica.

Fig. 1. Distribution over time of vascular plant collection events compiled for Isla del Coco, Puntarenas, Costa Rica.

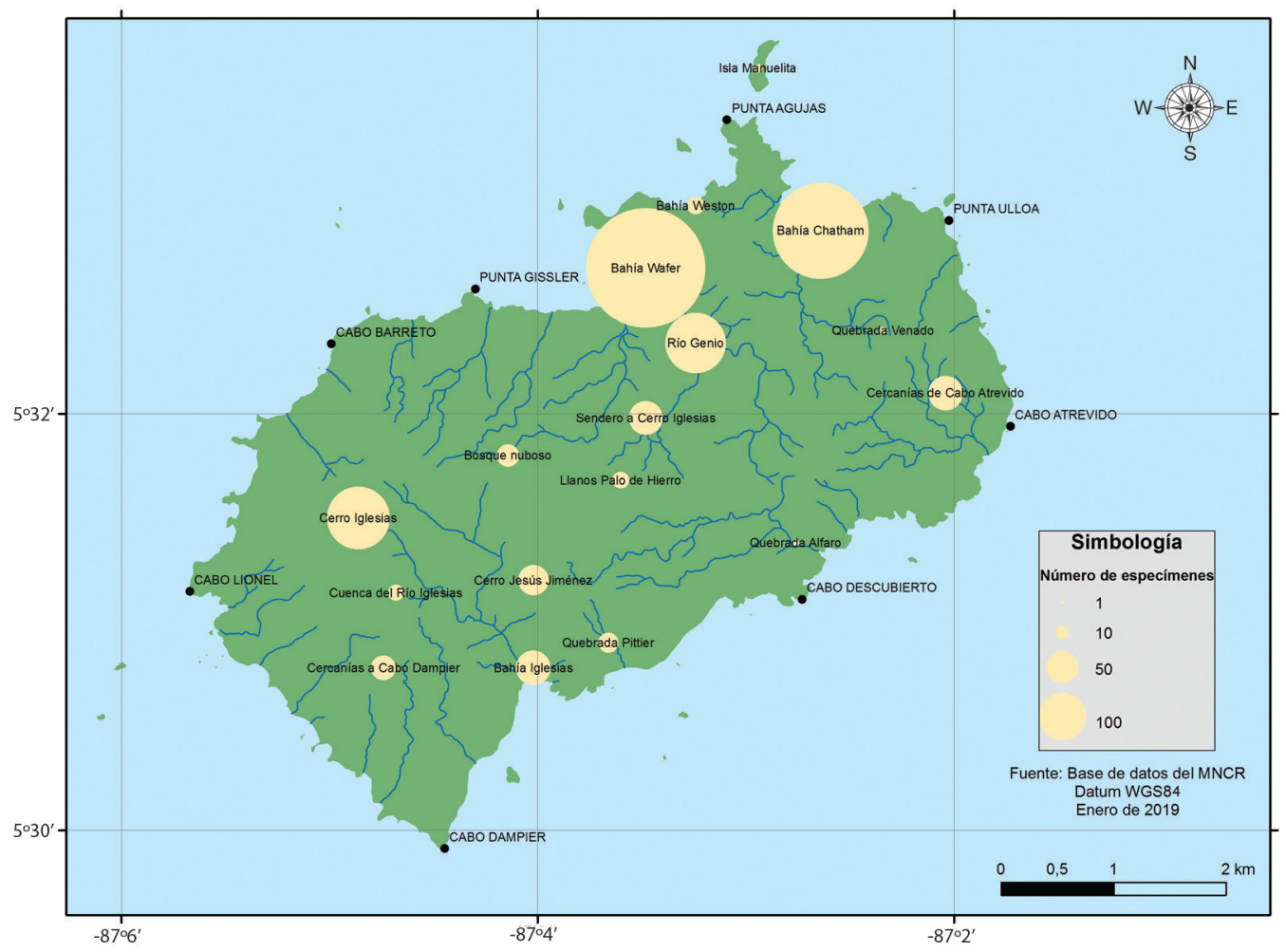

Fig. 2. Distribución del esfuerzo de recolecta de plantas vasculares en la Isla del Coco, Puntarenas, Costa Rica. Fig. 2. Distribution of sampling effort of vascular plants on Isla del Coco, Puntarenas, Costa Rica. 
isla, específicamente en los alrededores de las bahías Wafer (523 registros) y Chatham (331) y márgenes del río Genio (150), que incluyen un $56 \%$ de las recolectas. Otra área con cierto esfuerzo de muestreo se ubica en el sendero hacia Cerro Iglesias, que incluye un 14 \% (245) del total de registros. Las áreas menos recolectadas se ubican hacia el sur, sur-oeste y sur-este de la isla, sectores que apenas incluyen un 14 $\%$ (253) de las recolectas realizadas, en localidades como Bahía Iglesias (57), cuenca alta del río Iglesias (15, al norte de cabo Dampier (32), alrededores del cerro Jesús Jiménez (46), Llanos de Palo de Hierro (16), quebrada Pittier (22), quebrada Alfaro (4), quebrada Venado (4) y alrededores de cabo Atrevido (57). En un $16 \%$ de los datos no se especifica la localidad exacta de recolecta (Fig. 2).

Riqueza florística general: En la Isla del Coco se registra, hasta ahora, una riqueza florística total de 296 especies de plantas vasculares, pertenecientes a 199 géneros y 82 familias (ver apéndice). Estos datos representan un
$87 \%$ de la diversidad esperada, según el índice Chao-1, que estima 340 especies, o un $78 \%$ según la ecuación de Clench, que estima una diversidad de 378 especies (Fig. 3). Lo anterior indica un inventario bastante aceptable, aunque incompleto, producto de un esfuerzo de muestreo heterogéneo, concentrado en ciertas áreas y especies, pues solo un $22 \%$ de las especies poseen 10 o más registros y un $34 \%$ solo uno o dos.

De esta riqueza total, 219 especies son nativas $(74 \%)$ y $77(26 \%)$ son introducidas en la Isla del Coco. En el caso de las especies nativas, $117(53 \%)$ corresponden a angiospermas, $96(44 \%)$ a helechos y 6 a licófitos (3\%). Las especies nativas se agrupan mayormente en las familias Melastomataceae (15), Dryopteridaceae (13), Hymenophyllaceae (13), Thelypteridaceae (13), Fabaceae (12), Polypodiaceae (11), Rubiaceae (11) y Pteridaceae (9), que en conjunto corresponden un $44 \%$ (97 especies) de la diversidad de plantas nativas de la isla. Los géneros más diversos son Elaphoglossum (8), Thelypteris (8), Miconia

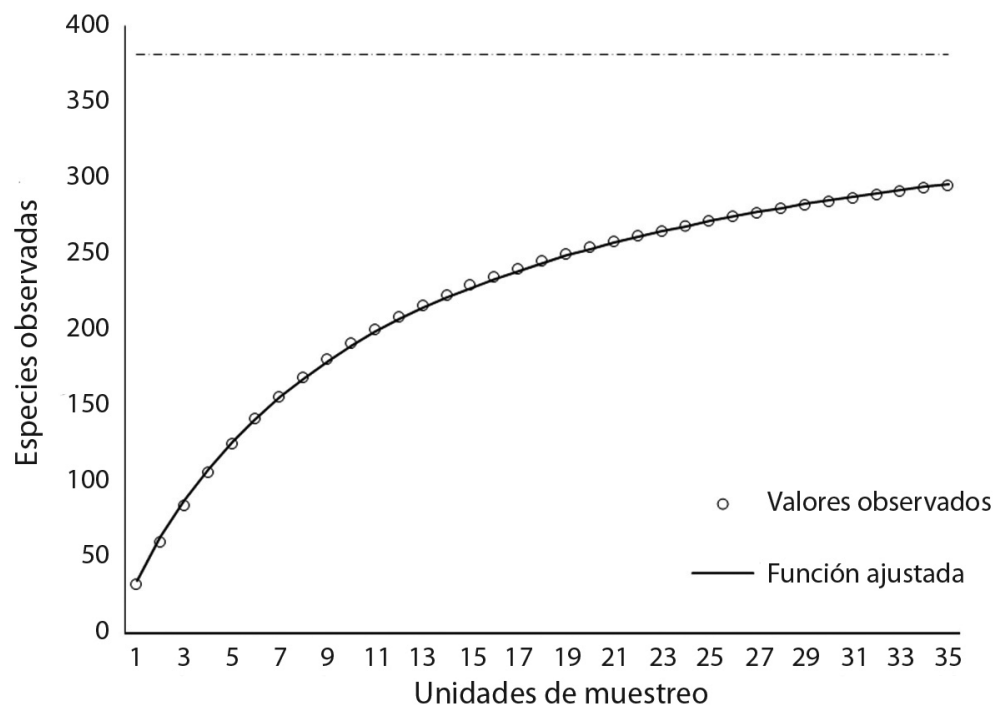

Fig. 3. Curva de acumulación de especies para el inventario de plantas recopilado de la Isla del Coco, Puntarenas, Costa Rica $\left(\mathrm{Sn}=79,48975 * \mathrm{n} /[1+[0,209958 * \mathrm{n}]] ;\right.$ Sobs. $=295 ;$ Sest. $[\mathrm{a} / \mathrm{b}]=378 ; \mathrm{R}^{2}=0,99967 ; 100$ aleatorizaciones $) .1$ unidad de muestreo $=103$ ejemplares.

Fig. 3. Species accumulation curve for the inventory of plants compiled for Isla del Coco, Puntarenas, Costa Rica ( $\mathrm{Sn}=$ $79,48975^{*} \mathrm{n} /\left[1+\left[0,209958^{*} \mathrm{n}\right]\right]$; Sobs. $=295 ;$ Sest. $[\mathrm{a} / \mathrm{b}]=378 ; \mathrm{R}^{2}=0,99967 ; 100$ aleatorizaciones). 1 sample unit $=103$ specimens. 
(12 spp.), Asplenium (5) e Ipomoea (5). Con base en el número de registros disponibles, como medida indirecta de abundancia, entre las especies nativas más comunes o abundantes están Marcgravia waferi (27 registros), Sacoglottis holdridgei (26), Phlegmariurus pittieri (25), Miconia strigillosa (22), Miconia lasiopoda (22), Ardisia compressa (21), Hypolytrum amplum (21), Ocotea insularis (21), Peperomia glabella (21), Miconia solearis (19), Trichomanes galeottii (19), Vandenboschia collariata (19) y Vittaria graminifolia (19).

En relación con las especies introducidas, la proporción registrada (26\%) es relativamente baja en comparación con otras islas océanicas del Pacífico, en donde se han registrado proporciones de especies introducidas similares o mayores a la de especies nativas (Lavergne, Rameau \& Figier, 1999; Trusty et al., 2006). No obstante, y a pesar de su baja proporción relativa, la presencia de plantas introducidas es un tema que merece especial atención, ya que algunas de estas especies podrían llegar a convertirse en invasoras y generar problemas de conservación para las especies nativas (Trusty et al., 2006; Lonsdale, 1999), situación que en la Isla del Coco aún no se observa de una forma considerable. Todas las especies introducidas de la isla son angiospermas y se agrupan principalmente en las familias Cyperaceae (12 spp.), Poaceae (10), Euphorbiaceae (4), Malvaceae (4), Rubiaceae (4), Asteraceae (3) y Plantaginaceae (3) y entre las especies más comunes están Rugoloa polygonata (14 registros), Hyptis capitata (11), Spermacoce exilis (10), Rhynchospora polyphylla (10), Paspalum nutans (9), Urena lobata var. lobata (8), Kyllinga brevifolia (8) y Diodia sarmentosa (8).

El endemismo en la isla corresponde a un $22 \%$ (48 spp.) de la flora nativa (219 spp.), lo cual es relativamente bajo comparado con otras islas o archipiélagos en el Pacífico, donde se registran porcentajes de un $29 \%$ a un $97 \%$ de endemismo (para más detalles ver Trusty et al., 2006), aunque mayor que el dato de endemismo continental, de alrededor de un $11 \%$ de la flora vascular de Costa Rica (Hammel et al., 2004). El pequeño tamaño de la isla, su relativa homogeneidad ambiental y su formación reciente, pueden influir en este porcentaje de endemismo y sus patrones de especiación, los cuales derivan de eventos de colonización independiente más que de especiación por eventos de radiación in situ (Igea, Bogarín, Papadopulos, \& Savolainen; 2015, Trusty et al., 2006).

La mayor parte de las especies endémicas son helechos (58\%, 28 spp.), y en menor grado angiospermas (38\%, 18 spp.) y licófitos (4\%, 2 spp). Las familias que agrupan mayor número de especies endémicas son Dryopteridaceae (7 spp.), Cyatheaceae (4), Hymenophyllaceae (3), Orchidaceae (3), Rubiaceae (3) y Thelypteridaceae (3). Los géneros con mayor representación de estas especies son Elaphoglossum (5 spp.), Cyathea (4) y Epidendrum (3). Entre las especies endémicas más comunes están Marcgravia waferi (27 registros), Sacoglottis holdridgei (26), Phlegmariurus pittieri (25), Vandenboschia collariata (19), Eugenia pacifica (18), Epidendrum cocoense (16) y Psychotria cocosensis (15).

La alta proporción de helechos endémicos (58\%), aunado su alta diversidad en general (44\%), en relación a la flora nativa, es un elemento distintivo de la composición florística de la Isla del Coco en comparación con otras islas oceánicas (Trusty et al., 2006), favorecido en mucho por la alta precipitación pluvial que recibe la isla, que en promedio varía entre los 5000 y $7000 \mathrm{~mm}$ anuales (Herrera, 1985). En el territorio continental, a pesar de su alta diversidad, los helechos representan un porcentaje mucho menor (12\%) de la flora vascular de Costa Rica (Hammel et al., 2004).

Riqueza florística por formas de plantas: La flora de la Isla del Coco tiene un componente muy alto de especies herbáceas, con un $63 \%$ del total, seguido en una proporción mucho menor, por las especies arbustivas, con un $16 \%$. Las formas menos representadas son la arborescente y los bejucos y enredaderas con un $12 \%$ y $8 \%$, respectivamente. Esta baja representación arbórea (12\%) es también un elemento característico de la composición 
florística de esta isla, asociado con la alta dominancia de la especie Sacoglottis holdridgei en los bosques (Acosta-Vargas, 2016). En el territorio continental, el componente arbóreo alcanza una proporción mayor de un 19\% de la flora vascular de Costa Rica (Hammel et al., 2004).

La diversidad de familias botánicas por forma de las plantas se muestra en la Fig. 4. En el componente herbáceo predominan las familias Cyperaceae y Poaceae, con 19 y 15 spp. respectivamente, seguidas por varias familias de helechos (Dryopteridaceae, Hymenophyllaceae, Thelypteridaceae, Polypodiaceae, Pteridaceae). Los géneros más diversos son Elaphoglossum (8 spp.), Thelypteris (8), Cyperus (7) y Asplenium (5). Entre las especies más comunes están Phlegmariurus pittieri $(25$ registros), Hypolytrum amplum (21), Peperomia glabella (21), Trichomanes galeottii (19), Vandenboschia collariata (19) y Vittaria graminifolia (19).
En el componente arbustivo destacan las familias Melastomataceae (14 spp.), Rubiaceae (8) y Malvaceae (5). Entre los géneros más diversos están Miconia (12 spp.) y Henriettea (2) y entre las especies más comunes están Marcgravia waferi (27 registros), Miconia strigillosa (22), Miconia lasiopoda (22), Ardisia compressa (21) y Miconia solearis (19).

El componente arborescente, está constituido por varias familias con pocas especies, entre las que destacan Cyatheaceae (4 spp.), Moraceae (3), Anacardiaceae, Annonaceae, Arecaceae, Combretaceae, Lauraceae, Primulaceae y Rutaceae (todas estas familias con 2 spp.). Los géneros más diversos son Cyathea (4 spp.), Annona (2), Citrus (2) y Ficus (2). Entre las especies más comunes están Sacoglottis holdridgei (26 registros), Ocotea insularis (21), Cyathea notabilis (13), Cecropia pittieri (13), Cyathea alfonsoana (12) y Cyathea nesiotica (11).
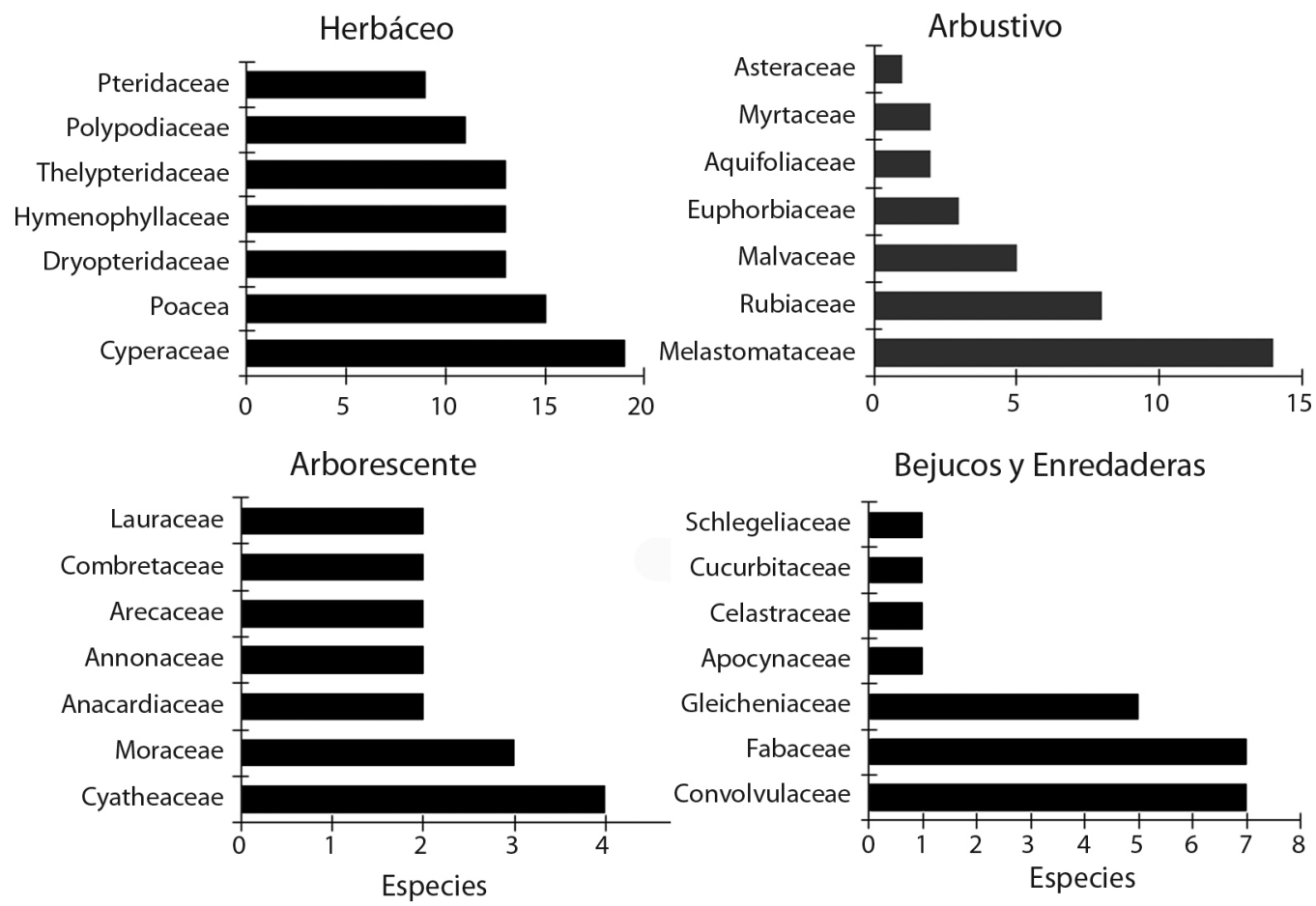

Fig. 4. Familias con mayor diversidad de especies según forma de las plantas en la Isla del Coco, Puntarenas, Costa Rica. Fig. 4. Families with a greater diversity of species according to plants form on Isla del Coco, Puntarenas, Costa Rica. 
Entre los bejucos y enredaderas predominan las familias Convolvulaceae (7 spp.), Fabaceae (7) y Gleicheniaceae (5). Los géneros más diversos son Ipomoea (6 spp.), Sticherus (3) y Mucuna (2). Entre las especies más comunes están Schlegelia brachyantha (11 reg.), Entada gigas (10), Gleichenella pectinata (6) y Tassadia obovata (6).

En la Isla del Coco habitan 58 especies epífitas de varias de las formas anteriores, que representan un $20 \%$ del total de la flora. La mayor parte de estas especies epífitas son helechos (69\%, 40 spp.), y en menor grado angiospermas $(28 \%, 16$ spp.) y licófitos $(3 \%$, 2 spp.). Este porcentaje es similar al dato continental de epífitas de un $22 \%$ de la flora vascular de Costa Rica (Hammel et al., 2004). El epifitismo es favorecido por la nubosidad presente en varios sectores de la isla y las fuertes precipitaciones pluviales durante la mayor parte del año. Las tres familias que agrupan más especies epífitas, son también de helechos: Polypodiaceae (11 spp.), Dryopteridaceae (10), Hymenophyllaceae (10), seguidas por Orchidaceae (5), Araceae (3) y Aspleniaceae (3). Los géneros más diversos son Elaphoglossum
(7 spp.), Asplenium (3), Didymoglossum (3), Epidendrum (3), Hymenophyllum (3), Polybotrya (3) y Trichomanes (3). Entre las especies epífitas más comunes están Marcgravia waferi (27 registros), Phlegmariurus pittieri (25), Peperomia glabella (21), Vittaria graminifolia (19), Campyloneurum phyllitidis (18) y Epidendrum cocoense (16).

Nuevos aportes taxonómicos: El conocimiento de la flora de la Isla del Coco se ha incrementado a través del tiempo con los estudios florísticos realizados (Rose, 1892; Robinson, 1902; Stewart, 1912; Svenson, 1935, 1938; Fosberg \& Klawe, 1966; Trusty et al., 2006, Fig. 5). En comparación con estos estudios, la presente lista incluye 45 especies nuevas para la isla (Tabla 1), de las cuales 16 son especies nuevas para la ciencia y endémicas para la isla, ocho son introducidas y cinco se identificaron solo a nivel de género. Las especies nuevas para la ciencia incluyen 12 especies de helechos y cuatro especies de angiospermas (Tabla 1).

Con respecto al inventario florístico más reciente previo a este trabajo (Trusty et al.,

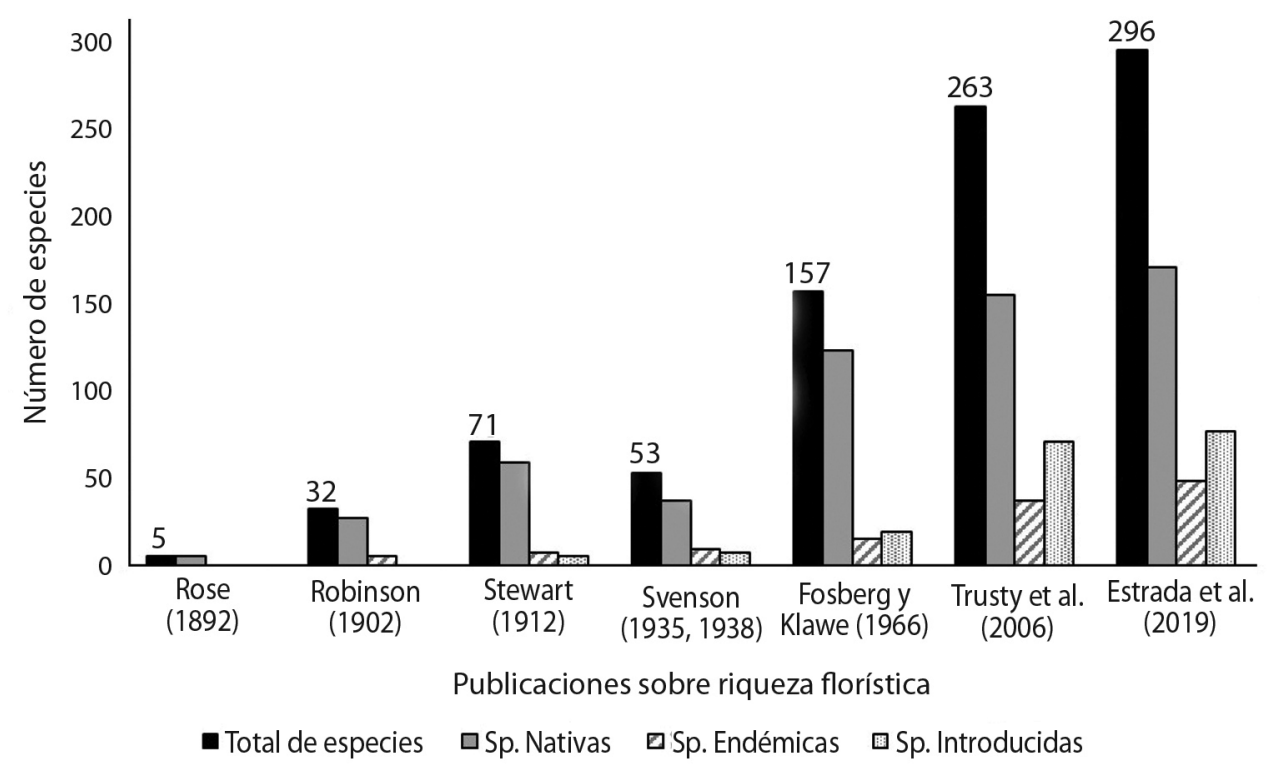

Fig. 5. Conocimiento de la flora, a través del tiempo en la Isla del Coco, Puntarenas, Costa Rica.

Fig. 5. Knowledge of the flora, through time and on Isla del Coco, Puntarenas, Costa Rica. 
TABLA 1

Lista de nuevos registros de especies de plantas vasculares para la Isla del Coco, Puntarenas, Costa Rica

TABLE 1

List of new records of vascular plants species from Isla del Coco, Puntarenas, Costa Rica

\begin{tabular}{|c|c|c|c|}
\hline Division & Familia & Especie & Observaciones \\
\hline Lycophyta & Selaginellaceae & Selaginella porelloides (Lam.) Spring (L.D. Gómez 6563, CR) & \\
\hline Monilophyta & Aspleniaceae & Asplenium cuspidatum Lam. (A. Rojas 8964, CR) & \\
\hline Monilophyta & Blechnaceae & Blechnum longistipitatum A. Rojas (A. Rojas 8187, CR) & $\begin{array}{l}\text { Nueva para la ciencia y } \\
\text { endémica (Rojas, 2011) }\end{array}$ \\
\hline Monilophyta & Cyatheaceae & Cyathea $\times$ hybrida A. Rojas (A. Rojas 8985, CR) & $\begin{array}{l}\text { Nueva para la ciencia y } \\
\text { endémica (Rojas, 2017) }\end{array}$ \\
\hline Monilophyta & Dennstaedtiaceae & Pteridium caudatum (L.) Maxon (L.D. Gómez 18050, CR) & \\
\hline Monilophyta & Dryopteridaceae & Elaphoglossum alvaradoanum A. Rojas (A. Rojas, 3620 CR) & $\begin{array}{l}\text { Nueva para la ciencia y } \\
\text { endémica (Rojas, 2009) }\end{array}$ \\
\hline Monilophyta & Dryopteridaceae & Elaphoglossum longicrure $\mathrm{H}$. Christ (A. Rojas, $9005 \mathrm{CR}$ ) & \\
\hline Monilophyta & Dryopteridaceae & $\begin{array}{l}\text { Elaphoglossum } \times \text { morphohybridum A. Rojas } \\
(\text { A. Rojas } 9281, \mathrm{MO})\end{array}$ & $\begin{array}{l}\text { Nueva para la ciencia y } \\
\text { endémica (Rojas, 2019) }\end{array}$ \\
\hline Monilophyta & Dryopteridaceae & Polybotrya bipinnata A. Rojas (A. Rojas 8157, CR) & $\begin{array}{l}\text { Nueva para la ciencia y } \\
\text { endémica (Rojas, 2011) }\end{array}$ \\
\hline Monilophyta & Dryopteridaceae & Polybotrya insularis A. Rojas (A. Estrada, 5897) & $\begin{array}{l}\text { Nueva para la ciencia y } \\
\text { endémica (Rojas, 2007) }\end{array}$ \\
\hline Monilophyta & Hymenophyllaceae & Hymenophyllum abruptum Hook. (A. Rojas 8977, MO) & \\
\hline Monilophyta & Marattiaceae & Danaea epilithica A. Rojas (A. Rojas 3644, CR) & $\begin{array}{l}\text { Nueva para la ciencia y } \\
\text { endémica (Rojas, 2013b) }\end{array}$ \\
\hline Monilophyta & Nephrolepidaceae & Nephrolepis cocosensis A. Rojas (A. Rojas 8191, CR), & $\begin{array}{l}\text { Nueva para la ciencia y } \\
\text { endémica (Rojas, 2017) }\end{array}$ \\
\hline Monilophyta & Polypodiaceae & Lellingeria vargasiana A. Rojas (Rojas 8998, CR) & $\begin{array}{l}\text { Nueva para la ciencia y } \\
\text { endémica (Rojas, 2011) }\end{array}$ \\
\hline Monilophyta & Polypodiaceae & Stenogrammitis grammitoides A. Rojas (A. Rojas, 8946, MO) & $\begin{array}{l}\text { Nueva para la ciencia y } \\
\text { endémica (Rojas, 2013a) }\end{array}$ \\
\hline Monilophyta & Pteridaceae & $\begin{array}{l}\text { Adiantum } \times \text { variopinnatum Jermy et T. G. Walker } \\
(\text { J. Valerio } 2224, \mathrm{CR})\end{array}$ & \\
\hline Monilophyta & Pteridaceae & Adiantum pulverulentum L. (A. Rojas 9275, CR) & \\
\hline Monilophyta & Tectariaceae & Tectaria $\times$ epilithica A. Rojas (A. Rojas 8211, CR) & $\begin{array}{l}\text { Nueva para la ciencia y } \\
\text { endémica (Rojas, 2017) }\end{array}$ \\
\hline Monilophyta & Thelypteridaceae & Thelypteris sp (L.D. Gómez 3361, CR) & \\
\hline Monilophyta & Thelypteridaceae & Thelypteris dentata (Forssk.) E. P. St. John (A. Rojas 8168, CR) & \\
\hline Monilophyta & Thelypteridaceae & $\begin{array}{l}\text { Thelypteris eggersii (Hieron.) C. F. Reed } \\
\text { (L. D. Gómez, 3326, CR) }\end{array}$ & \\
\hline Monilophyta & Thelypteridaceae & Thelypteris nana A. Rojas (A. Rojas 8940, CR) & $\begin{array}{l}\text { Nueva para la ciencia y } \\
\text { endémica (Rojas, 2011) }\end{array}$ \\
\hline Monilophyta & Thelypteridaceae & Thelypteris opulenta (Kaulf.) Fosberg (A. Rojas 8167, CR) & \\
\hline Spermatophyta & Amaranthaceae & Cyathula prostrata (L.) Blume (L. Acosta 15021, CR) & Introducida \\
\hline Spermatophyta & Annonaceae & Annona muricata L. (A. Estrada, Obs.) & Introducida \\
\hline Spermatophyta & Apocynaceae & Plumeria rubra L. (L.D. Gómez 3385, CR) & \\
\hline Spermatophyta & Araceae & Philodendron sagittifolium Liebm. - cf.- (R.B. Foster 4177, MO) & \\
\hline Spermatophyta & Araceae & $\begin{array}{l}\text { Spathiphyllum abelianum A. Rojas \& J.M. Chaves } \\
\text { (A. Rodríguez 14610, CR) }\end{array}$ & $\begin{array}{l}\text { Nueva para la ciencia y } \\
\text { endémica (Rojas \& } \\
\text { Chaves, 2011) }\end{array}$ \\
\hline Spermatophyta & Celastraceae & sp. (A. Rodríguez 14807, CR) & \\
\hline
\end{tabular}


TABLA 1 (Continuación) / TABLE 1 (Continued)

\begin{tabular}{|c|c|c|c|}
\hline Division & Familia & Especie & Observaciones \\
\hline Spermatophyta & Convolvulaceae & Ipomoea tiliacea (Willd.) Choisy (A. Jiménez 3185, CR) & \\
\hline Spermatophyta & Cyperaceae & Cyperus luzulae (L.) Rottb. ex Retz. (A. Rodríguez 14587, CR) & Introducida \\
\hline Spermatophyta & Cyperaceae & Eleocharis mutata (L.) Roem. \& Schult. (R. Soto s.n., USJ) & \\
\hline Spermatophyta & Cyperaceae & Fimbristylis littoralis Gaudich. (A. Rodríguez 14584, CR) & \\
\hline Spermatophyta & Fabaceae & Desmodium scorpiurus (Sw.) Desv. (R.B. Foster 4141, CR) & \\
\hline Spermatophyta & Gentianaceae & $\begin{array}{l}\text { Tachia blancoi. Al. Rodr. \& J. Sánchez-Gonz. } \\
\text { (A. Rodríguez14612, CR) }\end{array}$ & $\begin{array}{l}\text { Nueva para la ciencia y } \\
\text { endémica (Sánchez-González } \\
\text { \& Rodríguez, 2017) }\end{array}$ \\
\hline Spermatophyta & Linderniaceae & Lindernia crustacea (L.) F. Muell. (A. Rodríguez 14568, CR) & Introducida \\
\hline Spermatophyta & Melastomataceae & Miconia cocoensis Almeda \& Kriebel (J. Trusty 270, CR) & $\begin{array}{l}\text { Nueva para la ciencia y } \\
\text { endémica (Kriebel \& } \\
\text { Almeda, 2012) }\end{array}$ \\
\hline Spermatophyta & Melastomataceae & Miconia diegogomezii Kriebel \& Almeda (J. Trusty 68, CR) & $\begin{array}{l}\text { Nueva para la ciencia y } \\
\text { endémica (Kriebel \& } \\
\text { Almeda, 2012) }\end{array}$ \\
\hline Spermatophyta & Melastomataceae & Miconia sp1 (A. Estrada 5917, CR) & \\
\hline Spermatophyta & Melastomataceae & Miconia sp2 (A. Estrada 5899, CR) & \\
\hline Spermatophyta & Onagraceae & Oenothera rosea L'Her. ex Aiton (L.D. Gómez 3392, CR) & Introducida \\
\hline Spermatophyta & Plantaginaceae & Mecardonia procumbens (Mill.) Small (R.B. Foster 4142, MO) & Introducida \\
\hline Spermatophyta & Plantaginaceae & Stemodia angulata Oerst. (A. Rodríguez 14570, CR) & Introducida \\
\hline Spermatophyta & Rubiaceae & Palicourea sp (L. Holdridge 5168, F) & \\
\hline Spermatophyta & Verbenaceae & Verbena litoralis Kunth (L.D. Gómez 3390, CR) & Introducida \\
\hline
\end{tabular}

2006), se presenta un aumento en el número de especies (de 263 especies a 296), y el consecuente aumento de especies endémicas (37 a 48) e introducidas (71 a 77, Fig. 5). Se actualizaron o corrigieron 36 nombres de especies (Tabla 2), entre los que destacan ocho entidades que, aunque informadas en dicho estudio bajo un nombre específico o a nivel de género, se describieron posteriormente como especies nuevas para la ciencia (Tabla 2) y cinco casos en los cuales los nombres usados por Trusty et al. (2006), no se reconocen, pero aún no hay certeza de su identidad taxonómica (entidades a nivel de género).

En el presente catálogo se incluyen 10 especies cuya identidad taxonómica es incierta hasta ahora (identificadas a nivel de familia o género), que en su mayoría fueron registradas bajo algún nombre en publicaciones previas (Chaves, 2011; Trusty et al., 2006; Fosberg \& Klawe, 1966; Stewart, 1912), pero en el presente trabajo no se reconoce dicho nombre. Estas entidades son una Celastraceae (recolectada durante este estudio e identificada solo a nivel de familia), Miconia sp.2 (identificada como C. hirta por Fosberg \& Klawe, 1966 y Stewart, 1912), Ficus sp. (como Brosimum sp. por Trusty et al., 2006, Fosberg \& Klawe, 1966 y Svenson, 1935), Freziera sp. (como F. calophylla por Trusty et al., 2006), Hedyosmum sp. (como H. racemosum por Trusty et al., 2006), Ilex sp 1 (como I. yurumanguinis por Trusty et al., 2006), Ilex sp 2 (como I. yurumanguinis por Trusty et al., 2006), Miconia sp.1 (como M. chrysophylla por Chaves, 2011), Palicourea sp. (como Psychotria sp. A. por Taylor, 2014) y Thelypteris sp. (como T. quadrangularis por Gómez, 1975 b).

También existen nombres de especies incluidos en algunas publicaciones sobre la Isla del Coco, pero que no ha sido posible ubicar un ejemplar testigo para verificar su presencia y su correcta determinación, razón por la cual se han excluido del actual catálogo de especies y se consideran como datos dudosos. Estos nombres son: Acrostichum caudatum Cav. (Fosberg \& 
TABLA 2

Cambios nomenclaturales respecto a Trusty et al. (2006), Isla del Coco (Puntarenas, Costa Rica)

TABLE 2

Nomenclatural changes compared to Trusty et al. (2006), Isla del Coco, Puntarenas, Costa Rica

\begin{tabular}{|c|c|c|c|c|}
\hline División & Familia & Nombre aceptado & $\begin{array}{l}\text { Nombre (s) sensu } \\
\text { Trusty et al. (2006) }\end{array}$ & Observaciones \\
\hline Monilophyta & Dryopteridaceae & $\begin{array}{l}\text { Elaphoglossum } \\
\text { alvaradoanum A. Rojas }\end{array}$ & $\begin{array}{l}\text { Elaphoglossum auripilum var. } \\
\text { longipilosum Atehortúa }\end{array}$ & Especie nueva (Rojas, 2009) \\
\hline Monilophyta & Dryopteridaceae & $\begin{array}{l}\text { Elaphoglossum rojasii } \\
\text { N.T.L. Pena }\end{array}$ & Elaphoglossum reptans A. Rojas & $\begin{array}{l}\text { Reemplazo de nombre por } \\
\text { homonimia (Pena, Freitas \& } \\
\text { Alves-Araújo, 2017) }\end{array}$ \\
\hline Monilophyta & Dryopteridaceae & $\begin{array}{l}\text { Megalastrum galeottii } \\
\text { (M.Martens) R.C. Moran } \\
\text { \& J. Prado }\end{array}$ & $\begin{array}{l}\text { Megalastrum subincisum (Willd.) } \\
\text { A.R. Sm. \& R.C. Moran }\end{array}$ & \\
\hline Monilophyta & Dryopteridaceae & $\begin{array}{l}\text { Polybotrya bipinnata } \\
\text { A. Rojas }\end{array}$ & $\begin{array}{l}\text { Polybotrya polybotryoides (Baker) } \\
\text { H. Christ (pro parte) }\end{array}$ & Especie nueva (Rojas, 2011b) \\
\hline Monilophyta & Dryopteridaceae & $\begin{array}{l}\text { Polybotrya insularis } \\
\text { A. Rojas }\end{array}$ & $\begin{array}{l}\text { Polybotrya osmundacea Humb. \& } \\
\text { Bonpl. ex Willd. (pro parte) }\end{array}$ & Especie nueva (Rojas, 2007) \\
\hline Monilophyta & Dryopteridaceae & $\begin{array}{l}\text { Polybotrya polybotryoides } \\
\text { (Baker) H. Christ }\end{array}$ & $\begin{array}{l}\text { Polybotrya osmundacea Humb. \& } \\
\text { Bonpl. ex Willd. (pro parte) }\end{array}$ & \\
\hline Monilophyta & Hymenophyllaceae & $\begin{array}{l}\text { Polyphlebium capillaceum } \\
\text { (L.) Ebihara \& Dubuisson }\end{array}$ & $\begin{array}{l}\text { Trichomanes capillaceum var. } \\
\text { cocos (H. Christ) L.D. Gómez }\end{array}$ & $\begin{array}{l}\text { No se indica la variedad, ya } \\
\text { que aún no se formaliza dentro } \\
\text { del género Polyphlebium. }\end{array}$ \\
\hline Monilophyta & Hymenophyllaceae & $\begin{array}{l}\text { Vandenboschia collariata } \\
\text { (Bosch) Ebihara \& K. Iwats. }\end{array}$ & $\begin{array}{l}\text { Trichomanes collariatum Bosch } \\
\text { var. alvaradoi }\end{array}$ & $\begin{array}{l}\text { No se indica la variedad, ya } \\
\text { que aún no se formaliza dentro } \\
\text { del género Vandenboschia. }\end{array}$ \\
\hline Monilophyta & Marattiaceae & Danaea epilithica A. Rojas & Danaea nodosa (L.) Sm. & Especie nueva (Rojas, 2013) \\
\hline Monilophyta & Nephrolepidaceae & $\begin{array}{l}\text { Nephrolepis cocosensis } \\
\text { A. Rojas }\end{array}$ & $\begin{array}{l}\text { Nephrolepis biserrata (Sw.) Schott } \\
\text { (pro parte); Nephrolepis rivularis } \\
\text { (Vahl) Mett. Ex Krug }\end{array}$ & Especie nueva (Rojas, 2017) \\
\hline Monilophyta & Tectariaceae & $\begin{array}{l}\text { Tectaria moranii } \\
\text { Li Bing Zhang \& G.D. Tang }\end{array}$ & Tectaria incisa Cav. (pro parte) & $\begin{array}{l}\text { Especie nueva } \\
\text { (Tang \& Zhang, 2018) }\end{array}$ \\
\hline Spermatophyta & Aquifoliaceae & Ilex sp.1 & Ilex yurumanguinis Cuatrec. & \\
\hline Spermatophyta & Aquifoliaceae & Ilex sp.2 & Ilex yurumanguinis Cuatrec. & \\
\hline Spermatophyta & Chloranthaceae & Hedyosmum sp. & $\begin{array}{l}\text { Hedyosmum racemosum } \\
\text { (Ruiz \& Pav.) G. Don }\end{array}$ & \\
\hline Spermatophyta & Cyperaceae & Cyperus esculentus $\mathrm{L}$. & Cyperus sphacelatus Rottb. & \\
\hline Spermatophyta & Cyperaceae & $\begin{array}{l}\text { Cyperus hermaphroditus } \\
\text { (Jacq.) Standl. }\end{array}$ & Cyperus tenuis Sw. & \\
\hline Spermatophyta & Cyperaceae & $\begin{array}{l}\text { Rhynchospora radicans } \\
\text { subsp. microcephala (Bertero } \\
\text { ex Spreng.) W. W. Thomas }\end{array}$ & $\begin{array}{l}\text { Rhynchospora pubera subsp. } \\
\text { parvula (Vahl) Boeck. }\end{array}$ & \\
\hline Spermatophyta & Fabaceae & $\begin{array}{l}\text { Dalbergia brownei } \\
\text { (Jacq.) Urb. }\end{array}$ & Dalbergia monetaria L. f. & \\
\hline Spermatophyta & Fabaceae & Dioclea reflexa Hook. f. & Canavalia maritima Thouars & \\
\hline Spermatophyta & Fabaceae & Mucuna urens (L.) DC. & Mucuna mutisiana (Kunth) DC. & \\
\hline Spermatophyta & Malvaceae & Sida hirsutissima Mill. & Sida acuta Burm. f. & \\
\hline Spermatophyta & Melastomataceae & $\begin{array}{l}\text { Miconia alternidomatia } \\
\text { Michelang. }\end{array}$ & Maieta poeppigii Mart. ex Cogn. & \\
\hline Spermatophyta & Melastomataceae & $\begin{array}{l}\text { Miconia appendiculata } \\
\text { Triana }\end{array}$ & Miconia prasina (Sw.) DC. & \\
\hline
\end{tabular}


TABLA 2 (Continuación) / TABLE 2 (Continued)

\begin{tabular}{|c|c|c|c|c|}
\hline División & Familia & Nombre aceptado & $\begin{array}{l}\text { Nombre (s) sensu } \\
\text { Trusty et al. (2006) }\end{array}$ & Observaciones \\
\hline Spermatophyta & Melastomataceae & $\begin{array}{l}\text { Miconia cocoensis } \\
\text { Almeda \& Kriebel }\end{array}$ & Miconia sp. A & $\begin{array}{l}\text { Especie nueva (Kriebel \& } \\
\text { Almeda, 2012) }\end{array}$ \\
\hline Spermatophyta & Melastomataceae & $\begin{array}{l}\text { Miconia diegogomezii } \\
\text { Kriebel \& Almeda }\end{array}$ & Miconia sp. B & $\begin{array}{l}\text { Especie nueva (Kriebel \& } \\
\text { Almeda, 2012) }\end{array}$ \\
\hline Spermatophyta & Melastomataceae & $\begin{array}{l}\text { Miconia lasiopoda (Benth.) } \\
\text { Michelang. }\end{array}$ & Conostegia lasiopoda Benth. & \\
\hline Spermatophyta & Melastomataceae & Miconia silviphila Michelang. & Clidemia ombrophila Gleason & \\
\hline Spermatophyta & Melastomataceae & $\begin{array}{l}\text { Miconia strigillosa }(\mathrm{Sw} .) \\
\text { Judd \& Ionta }\end{array}$ & Clidemia strigillosa (Sw.) DC. & \\
\hline Spermatophyta & Meliaceae & Guarea microcarpa C. DC. & Guarea glabra Vahl & \\
\hline Spermatophyta & Moraceae & Ficus sp. & Brosimum sp. & \\
\hline Spermatophyta & Pentaphylacaceae & Freziera sp. & $\begin{array}{l}\text { Freziera calophylla Triana \& } \\
\text { Planch. }\end{array}$ & \\
\hline Spermatophyta & Poaceae & Paspalum nutans Lam. & Paspalum decumbens Sw. & \\
\hline Spermatophyta & Poaceae & $\begin{array}{l}\text { Rugoloa polygonata } \\
\text { (Schrad.) Zuloaga }\end{array}$ & $\begin{array}{l}\text { Panicum polygonatum Schrad.; } \\
\text { Panicum laxum } \mathrm{Sw} .\end{array}$ & \\
\hline Spermatophyta & Rhizophoraceae & Cassipourea killipii Cuatrec. & Cassipourea guianensis Aubl. & \\
\hline Spermatophyta & Rubiaceae & Spermacoce alata Aubl. & Borreria ocymoides (Burm. f.) DC. & \\
\hline Spermatophyta & Rubiaceae & Spermacoce remota Lam. & Borreria prostrata (Aubl.) Miq. & \\
\hline
\end{tabular}

Klawe, 1966 y Robinson, 1902), Anthephora hermaphrodita (L.) Kuntze (Morales, 2003), Ardisia humilis Vahl. (Fosberg \& Klawe, 1966 y Stewart, 1912), Asplenium cristatum Lam. (Fosberg \& Klawe, 1966), Caesalpinia eriostachys Benth. (Trusty et al., 2006, con base en una recolecta de Barclay, pero según se indica quizás puede haber un error de etiquetado), Clidemia bullosa DC. (Fosberg \& Klawe, 1966), Dennstaedtia ordinata (Kaulf.) T. Moore (Fosberg \& Klawe, 1966), Lippia origanoides Kunth (Rueda \& Hammel, 2015), Lycopodium mollicomum (Spring) Spring (Fosberg \& Klawe, 1966), Melinis minutiflora P. Beauv. (Fosberg \& Klawe, 1966), Nephrodium pectinatum (Willd.) Link (Rose, 1892), Nephrolepis pectinata (Willd.) Schott (Fosberg \& Klawe, 1966 y Stewart, 1912), Ossaea quinquenervia (Mill.) Cogn. (Fosberg \& Klawe 1966), Paspalum vaginatum Sw. (Rose, 1892), Pharus vittatus L. (Morales, 2003), Polystichum adiantiforme (G. Forst.) J. Sm. (Fosberg \& Klawe, 1966 y Stewart, 1912), Schwackaea cupheoides (Benth.) Cogn. (Trusty et al., 2006), Selaginella galeottii Spring (Stewart,
1912), Selaginella stenophylla A. Braun (Fosberg \& Klawe, 1966) y Robinson, 1902), Setaria setosa (Sw.) P. Beauv (Fosberg \& Klawe, 1966 y Stewart, 1912), Trichomanes prieurii Kunze (Fosberg \& Klawe, 1966 y Robinson, 1902), Trichomanes pyxidiferum L. (Fosberg \& Klawe, 1966 y Robinson, 1902), Turnera ulmifolia L. (González, 2015).

Finalmente, un grupo de especies introducidas se excluyó del presente catálogo porque se determinó que ya no se encuentran en la isla: Annona cherimola Mill., Cassia fistula L., Codiaeum variegatum (L.) Juss., Delonix regia (Bojer) Raf., Hyparrhenia rufa (Nees) Stapf, Nicotiana tabacum L., Portulaca oleracea L. y Senna reticulata (Willd.) H. S. Irwin \& Barneby.

Declaración de ética: los autores declaran que todos están de acuerdo con esta publicación y que han hecho aportes que justifican su autoría; que no hay conflicto de interés de ningún tipo; y que han cumplido con todos los requisitos y procedimientos éticos y legales pertinentes. Todas las fuentes de financiamiento 
se detallan plena y claramente en la sección de agradecimientos. El respectivo documento legal firmado se encuentra en los archivos de la revista.

\section{AGRADECIMIENTOS}

Al Área de Conservación Marina Isla del Coco (ACMIC) del Sistema Nacional de Áreas de Conservación (SINAC), del Ministerio de Ambiente y Energía (MINAE) por las facilidades brindadas y por permitirnos realizar esta investigación en el Parque Nacional Isla del Coco. A los funcionarios del Parque Nacional Isla del Coco por su colaboración durante los recorridos en el área, en especial a Guillermo Blanco Segura por su valioso apoyo en el campo y a Esteban Herrera Herrera por el apoyo logístico para la realización de las giras de campo. Al pteridólogo Robbin C. Moran, por su disposición y apoyo en atender las consultas realizadas sobre la taxonomía de los helechos de la isla. A los dos revisores anónimos por sus valiosos aportes, que permitieron mejorar el artículo. Finalmente, al Museo Nacional de Costa Rica por proporcionar el financiamiento y apoyo logístico a esta investigación. Este proyecto fue llevado a cabo mediante las resoluciones del SINAC: 2015-I-ACMIC-017, 2016-I-ACMIC-016, 18-2017-I-ACMC-018.

\section{RESUMEN}

Introducción: La flora de la Isla del Coco ha sido objeto de diversos esfuerzos de recolecta, estudios y publicaciones desde hace más de 300 años, hasta llegar a registrarse 263 especies; no obstante, una exploración reciente permitió determinar que se han presentado muchos cambios nomenclaturales, de clasificación y de incremento de especies desde la última publicación, lo cual hace necesario la publicación de una lista actualizada de especies. Objetivo: Elaborar un catálogo actualizado de las plantas vasculares de la Isla del Coco. Métodos: Mediante la búsqueda y revisión de ejemplares de herbario, publicaciones y recolecta de plantas entre 2016 y 2017, se elaboró una lista actualizada de plantas de la isla. Además, se determinó la riqueza estimada y la calidad del inventario utilizando estimadores de biodiversidad y la proporción de especies de acuerdo a su forma, hábitat epífito y procedencia. Resultados: Se registraron 296 especies de plantas vasculares, en 199 géneros y 82 familias, lo cual representa alrededor de un $80 \%$ de la diversidad esperada según los estimadores utilizados (Chao-1 y ecuación de Clench). Del total, 219 (74\%) especies son nativas y 77 (26\%) son introducidas. Las especies nativas incluyen angiospermas (53\%), helechos (44\%) y licófitos (3\%) y las introducidas solo angiospermas. Se indican las familias y géneros más diversos y las especies más comunes o abundantes. Se presenta un endemismo del 22\% (48 spp.) de la flora nativa, compuesto principalmente por helechos (58\%). La flora presenta una alta proporción de especies herbáceas (63\%) y una baja representación de especies arborescentes (12\%) y de bejucos y enredaderas ( $8 \%$ ). También se presenta una alta proporción de epífitas, con un $20 \%$ del total de la flora, compuesto mayormente por helechos (69\%). Este estudio adicionó 45 nuevas especies a la isla, de las cuales 16 son especies nuevas para la ciencia y endémicas para la isla y ocho son especies introducidas. También se actualizaron o corrigieron 36 nombres de especies en comparación con Trusty et al. (2006). Se proporciona un catálogo de especies con anotaciones. Conclusiones: La flora de la Isla del Coco presenta ciertas particularidades que la distinguen de otras islas oceánicas del Pacífico, en especial por la alta proporción de helechos endémicos y la alta diversidad de este grupo en general y el porcentaje relativamente bajo de endemismo. También es característico la baja diversidad de especies arbóreas, con una alta dominancia de Sacoglottis holdridgei en todos los bosques de la Isla y la alta proporción de especies epífitas, compuesto principalmente por helechos. La proporción de especies introducidas es relativamente baja en comparación con otras islas oceánicas del Pacífico.

Palabras clave: plantas vasculares, diversidad florística, islas oceánicas, Isla del Coco.

\section{REFERENCIAS}

Acosta-Vargas, L. (2016). Population status of the tree Sacoglottis holdridgei (Humiriaceae) at Isla del Coco National Park, Costa Rica. Revista de Biología Tropical, 64(Supplement 1), 263-275.

APG IV. (2016). An update of the Angiosperm Phylogeny Group classification for the orders and families of flowering plants: APG IV. Botanical Journal of the Linnean Society, 181(1), 1-20.

Belcher, E. (1843). Narrative of a voyage round the world, performed in her majesty's ship Sulphur during the years 1836-1842. Vol. 1. London, UK: Henry Colburn.

Bentham, G. (1844). The Botany of the Voyage of H.M.S. Sulphur, Under Command of Captain Sir Edward Belcher, During the Years 1836-42. London, UK: Smith, Elder \& Co. 
Bogarín, D., Warner, J., Powell, M., \& Savolainen, V. (2011). The orchid flora of Cocos Island National Park, Puntarenas, Costa Rica. Botanical Journal of the Linnean Society, 166, 20-39.

Chaves Fallas, J. M. (2011). Nuevo registro de Miconia chrysophylla (Rich.) Urb. (Melastomataceae) para la Isla del Coco, Costa Rica. Brenesia, 75-76, 101-102.

Colwell, R. K. (1997). EstimateS: Statistical estimation of species richness and shared species from samples. Versión 6.0b1. User's Guide and application published. Recuperado de http://viceroy.eeb.uconn.edu./ estimates.

Dampier. W. (1699). A new voyage round the world. IV edition corrected. Vol.1. London: The Crown in St. Pauls Church-yard. Recuperado de https://play.google.com/books/reader?id=adsNAAAAQAAJ\&hl=es $\&$ pg $=$ GBS.PP9

Decreto Ejecutivo 8748. La Gaceta Diario Oficial, San José, Costa Rica, 11 de julio de 1978.

Fosberg, F. R. \& Klawe, W. L. (1966). Preliminary list of plants from Cocos Island. En R. I. Bowman. (Ed.), The Galápagos. Proceedings of the Symposia of the Galápagos International Scientific Project (pp. 187-189). California, USA: University of California Press.

Gómez, L. D. (1975 a). The ferns and fern-allies of Cocos Island, Costa Rica . American Fern Journal, 65(4), $102-4$

Gómez, L. D. (1975 b). Contribuciones a la pteridología costarricense. VII. Pteridofitos de la Isla de Cocos. Brenesia, 6, 33-48.

González, J. (2015). Turneraceae. En B. E. Hammel, M H. Grayum, C. Herrera, \& N. Zamora (Eds.). Manual de Plantas de Costa Rica, vol. VIII. Dicotiledóneas: Sabiaceae-Zygophyllaceae. Monographs in Systematic Botany from the Missouri Botanical Garden, 131,459 .

Hammel, B. E., Grayum, M. H., Herrera, C., \& Zamora, N. (Eds.). (2004). Manual de Plantas de Costa Rica. vol. I. Introducción/Introduction. Monographs in Systematic Botany from the Missouri Botanical Garden, 97, 2.

Hammel, B. E., Grayum, M. H., Herrera, C., \& Zamora, N. (Eds.). (2003a). Manual de Plantas de Costa Rica, vol. II. Gimnospermas y Monocotiledóneas: Agavaceae-Musaceae. Monographs in Systematic Botany from the Missouri Botanical Garden, 92, 1-694.

Hammel, B. E., Grayum, M. H., Herrera, C., \& Zamora N. (Eds.). (2003b). Manual de Plantas de Costa Rica, vol. III. Monocotiledóneas: Orchidaceae-Zingiberaceae. Monographs in Systematic Botany from the Missouri Botanical Garden, 93, 1-884.
Hammel, B. E., Grayum, M. H., Herrera, C., \& Zamora, N. (Eds.). (2010). Manual de Plantas de Costa Rica, vol. VI. Dicotiledóneas: Clusiaceae-Gunneraceae. Monographs in Systematic Botany from the Missouri Botanical Garden, 119, 1-970.

Hammel, B. E., Grayum, M. H., Herrera, C., \& Zamora, N. (Eds.). (2007). Manual de Plantas de Costa Rica, vol. V. Dicotiledóneas: Haloragaceae-Phytolaccaceae. Monographs in Systematic Botany from the Missouri Botanical Garden, 111, 1-933.

Hammel, B. E., Grayum, M. H., Herrera, C., \& Zamora, N. (Eds.). (2014). Manual de Plantas de Costa Rica, vol. VII. Dicotiledóneas: Picramniaceae-Rutaceae Monographs in Systematic Botany from the Missouri Botanical Garden, 129, 1-840.

Hammel, B. E., Grayum, M. H., Herrera, C., \& Zamora, N. (Eds.). (2015). Manual de Plantas de Costa Rica, vol. VIII. Dicotiledóneas: Sabiaceae-Zygophyllaceae. Monographs in Systematic Botany from the Missouri Botanical Garden, 131, 1-657.

Herrera, W. (1985). Clima de Costa Rica. San José, Costa Rica: EUNED.

Igea, J., Bogarín, D., Papadopulos, A.S.T \& Savolainen, V. (2015). A comparative analysis of island floras challenges taxonomy-based biogeographical models of speciation. Evolution, 69(2), 482-491.

Instituto Geográfico Nacional de Costa Rica (IGNCR). (2000). Hoja Isla del Coco. Costa Rica. Escala 1: 25000 .

Jiménez-Valverde, A. \& Hortal, J. (2003). Las curvas de acumulación de especies y la necesidad de evaluar la calidad de los inventarios biológicos. Revista Ibérica de Aracnología, 8, 151-161.

Kriebel, R. \& Almeda, F. (2012). Five new species of Miconia (Melastomataceae: Miconieae) from Costa Rica and Panamá. Harvard Papers in Botany, 17(1), 53-64.

Lavergne, C., Rameau, J. C., \& Figier, J. (1999). The invasive woody weed Ligustrum robustum subsp. walkeri threatens native forests on La Réunion. Biological Invasions, 1, 377-392.

Lonsdale, W. M. (1999). Global patterns of plant invasions and the concept of invasibility. Ecology, 80, 1522-1536.

Morales, J. F. (2003). Poaceae. En B. E. Hammel, M. H. Grayum, C. Herrera, \& N. Zamora. Manual de Plantas de Costa Rica, vol. III. Monocotiledóneas: Orchidaceae-Zingiberaceae. Monographs in Systematic Botany from the Missouri Botanical Garden, 93, 598-821. 
Nivia Ruíz, A. \& Cascante Marín, A. (2008). Distribución de las formas de vida en la flora costarricense. Brenesia, 69, 1-17.

Pena, N. T. L., Freitas, J., \& Alves-Araújo, A. (2017). Elaphoglossum maya and E. rojasii (Dryopteridaceae), replacement names for $E$. angustifrons and E. reptans, respectively. Phytotaxa, 307(2), 159-160.

Pittier, H. (1899). Apuntamientos preliminares sobre la Isla de Cocos, posesión costarricense en el Océano Pacífico. Memoria de la Secretaría de Fomento 1898/1899. Costa Rica, San José: Tipografía Nacional.

Robinson, B. L. (1902). Flora of the Galápagos Islands. Contributions from the Gray Herbarium of Harvard University, new series, no. 24. Proceedings of the American Academy of Arts and Sciences, 38(4), 77-269.

Rojas Alvarado, A. F. (2007). Novedades en Polybotrya (Filicales: Dryopteridaceae) para Costa Rica. Lankesteriana, 7(3): 557-562.

Rojas Alvarado, A. F. (2009). Two new species and a new combination in Elaphoglossum sect. Polytrichia subsect. Apoda (Dryopteridaceae) from Costa Rica and Panama. Brittonia, 61(3), 293-300.

Rojas Alvarado, A. F. (2011). New species and new records of ferns (Pteridophyta: Polypodiales) from Cocos Island, Costa Rica. Brenesia, 75-76, 7-15.

Rojas Alvarado, A. F. (2013a). Novelties in Grammitis Sw. and Stenogrammitis Labiak (Polypodiaceae) from Costa Rica, Colombia, y Venezuela. Actualidades Biológicas, 35(98), 5-10.

Rojas Alvarado, A. F. (2013b). Notas taxonómicas en Danaea Sm. (Marattiaceae) para Costa Rica, Panamá y Colombia. Actualidades Biológicas, 35(98), 11-20.

Rojas Alvarado, A. F. (2017). A new species and three hybrids in the ferns from Cocos Island, Puntarenas, Costa Rica. Acta Botánica Malacitana, 42(1), 91-99.

Rojas Alvarado, A. F. (2019). Corrections in recently described species of ferns and Lycophytes from the Neotropics. Open Access Library Journal, 6(e5172), 1-7.

Rojas Alvarado, A. F., \& Trusty, J. (2004). Diversidad Pteridofítica de la Isla del Coco, Costa Rica. Brenesia, $62,1-14$.
Rojas-Alvarado, A. F., \& Chaves-Fallas, J. M. (2011). Una especie nueva de Spathiphyllum (Araceae) para Costa Rica. Brenesia, 75-76, 4-6.

Rose, J. N. (1892). List of plants from Cocos Island. Contributions from the United States National Herbarium, 1, 135 .

Rueda, R., \& Hammel, B. E. (2015). Verbenaceae. En B. E. Hammel, M. H. Grayum, C. Herrera, \& N. Zamora (Eds.). Manual de Plantas de Costa Rica, vol. VIII. Dicotiledóneas: Sabiaceae-Zygophyllaceae. Monographs in Systematic Botany from the Missouri Botanical Garden, 131, 573-574.

Sánchez-González, J., \& Rodríguez, A. (2017). Una especie nueva de Tachia (Gentianaceae) de Isla del Coco, Costa Rica. Journal of the Botanical Research Institute of Texas, 11(2), 328-333.

Soberón, J., \& Llorente, J. (1993). The use of species accumulation functions for the prediction of species richness. Conservation Biology, 7, 480-488.

Stewart, A. (1912). Expedition of the California Academy of Sciences to the Galápagos Islands. 1905-1906. V. Notes of the botany of Cocos Island. Proceedings of the California Academy of Sciences, 4th series, 1, 375-404.

Svenson, H. K. (1935). Plants of the Astor Expedition, 1930 (Galápagos and Cocos Islands). American Journal of Botany, 22, 208-277.

Svenson, H. K. (1938). Pteridophyta of the Galápagos and Cocos Islands. Bulletin of the Torrey Botanical Club, 65, 303-333.

Tang, G., \& Zhang, L. (2018). Tectaria moranii (Tectariaceae), a new fern species from Costa Rica. Phytotaxa, 357 (3), 230-234.

Taylor, C. M. (2014). Rubiaceae. En B. E. Hammel, M. H. Grayum, C. Herrera \& N. Zamora (Eds.). 2014. Manual de Plantas de Costa Rica, vol. VII. Dicotiledóneas: Picramniaceae-Rutaceae. Monographs in Systematic Botany from the Missouri Botanical Garden, 129, 464-779.

Trusty, J. L., Kesler, H. C., \& Haug Delgado, G. (2006). Vascular flora of Isla del Coco, Costa Rica. Bulletin of the Torrey Botanical Club, 57(7), 247-355.

See Digital Appendix at: / Ver Apéndice digital en:

revistas.ucr.ac.cr 